\title{
A Review on Modalities of Treatment of External Cervical Root
}

\section{Resorption}

\author{
Baharan Ranjbar 01*, Negar $\mathrm{S}^{2}$ and Ensiyeh $\mathbf{R}^{2}$ \\ ${ }^{1}$ Department of Operative Dentistry, Qazvin University of Medical Sciences, Iran \\ 2Post Graduate Student in Operative Dentistry, Qazvin University of Medical Sciences, \\ Iran
}

Mini Review

Volume 4 Issue 2

Received Date: March 04, 2019

Published Date: April 12, 2019

DOI: $10.23880 /$ oajds-16000221

*Corresponding author: Baharan Ranjbar Omidi, Assistant professor, Department of Operative Dentistry, Dental Caries Prevention Research Center, Qazvin University of Medical Sciences, Qazvin, Iran, Tel: +98912 6447687; Email: baharanomidi@yahoo.com

Keywords: External cervical resorption; Odontoclastic action; Theraputic modalities

Abbreviations: ECR: External Cervical Resorption; MICRR: Multiple Idiopathic Cervical Root Resorption; MTA: Mineral Trioxide Aggregate.

\section{Introduction}

External cervical resorption (ECR) describes the loss of dental hard tissue beginning at the cervical region of the root surface as a result of odontoclastic action [1]. Studies have shown a prevalence of post traumatic injuries to permanent teeth that range from $3.9 \%$ to $58.6 \%$ [2]. Among the types of injuries, the most frequent are pulp necrosis, internal and external resorption, calcific metamorphosis, and ankylosis [3]. There are several reasons for the high incidence of maxillary canine impaction. They include crowding; a narrow maxillary arch; a Class II, division 2-incisor relationship; familial tendency; follicular disturbance of the canine; and pathology to the primary predecessors [4-6]. Ectopic eruption of canines may cause severe root resorption of adjacent teeth [7].

\section{Material and Methods}

Narrative review on published articles written in English language only reporting data related to cervical root resorption. Comprehensive electronic search was performed using PubMed, Google scholar, furthermore a manual search was conducted in related journals. The search terms including cervical root resorption, theraputic modalites and prognosis. A total of 16 articles were reviewed carefully and thoroughly in order to extract the information regarding different case reports on cervical root resorption with an attempt to offer suggestion that clinicians could be used as guideline in the clinical practice in attempt to different theraputic modalites and prognosis.

\section{Discussion}

There are different modalities for treatment of external cervical root resorption. Treatment alternatives are case-dependent and depends on the etiology of resorption. The basic aim of treating is the complete removal of resorptive tissue and the restoration of the defected area.

Clarisse $\mathrm{CH} \mathrm{Ng}$, et al. reported that for restored endodontically treated teeth that do not have complete circumferential tooth structure between the core and preparation finish line, the location of the remaining coronal tooth structure may affect their fracture resistance [8].

Bryan $\mathrm{T}$ Harris had a clinical report presents the treatment of a maxillary central incisor with class III invasive cervical resorption and a compromised ferrule. The patient was satisfied with the tooth preservation, and no further complications were noted at the 30 -month follow-up [9]. 


\section{Open Access Journal of Dental Sciences}

Multidisciplinary Approach for the Treatment of Extensive External Cervical Resorption after Dental Trauma was reported by Alves TP, et al. This case followed conservative therapy for the tooth structure that remained after an extensive external cervical resorption and was associated with careful follow-up sessions. It is extremely important to preserve the injured tooth in young patients both from the psychologic aspect and to maintain function and esthetics [10].

Case report of Restoration and retention of maxillary anteriors with severe root resorption was presented by Rhonda $\mathrm{R}$, et al. They emphasized that increased tooth mobility and long-term retention are significant concerns in cases of patients who have severe root resorption. Attaining stability with lingual splinting can cause esthetic problems. An interdisciplinary approach is necessary to provide optimal care for the patient [11].

A case report on Invasive cervical resorption: treatment challenges by Yookyung Kim. This case presents an invasive cervical resorption that finally resulted in extraction of the tooth. It may have been caused by damage in cervical cementum, physically from avulsion and chemically from intracoronal bleaching procedure. Invasive cervical resorption in advanced stages may present great challenges for clinicians. Therefore, prevention and early detection must be stressed when dealing with patients presenting history of potential predisposing factors [12].

Bartok RI done a review of literature with a topic of External radicular resorption, selected cases and review of the literature and concluded that the treatment of inflammatory external root resorption might be predictable, depending on the etiology [13].

- Pulpal infection may perpetuate the resorptive process, being the most important stimulation factor for the root resorption.

- The non-surgical treatment of the inflammatory resorption has to reduce the bacterial content of the endodontic system and allow the healing of the periradicular space. In addition, an adequate debridement and disinfection-intracanalar medication with calcium hydroxide and additives (chlorhexidine 2\%) for 3-6 months was used [13].

Case Report on Multiple idiopathic cervical root resorptions, report of one case with 8 teeth involved successively by Yin-Hua Jiang suggested that the prediction and prevention of Multiple Idiopathic Cervical Root Resorption (MICRR) are still impossible, and precise diagnosis and treatment are often far from easy, depending on the severity and localization of the defect. The treatment of MICRR often involves surgical exposure of the invaded surface to completely remove the inflammatory tissues, but high failure rate was seen due to recurrence. Exposure of the resorption defect combined with ostectomy has also been advocated to reduce recurrence [14].

Leila Eftekhar, et al. reported a case on Management of Invasive Cervical Root Resorption in a mandibular Canine Using Biodentine as a Restorative Material, In the case presented the diagnosis was Heithersay's class III ICR. The treatment plan was combination of surgical approach and non-surgical root canal treatment. The selected material was Biodentine because of better consistency after mixing, better handling properties, faster setting and also less tooth discoloration, compared to mineral trioxide aggregate (MTA). It is bioactive, biocompatible, and non-resorbable and having sufficient amount of push out bond strength with dentinal walls which makes it an excellent material for perforation repair. Also it produces tighter seal in the area of the defect and prevents microleakage [15].

Another, modality of treatment was reported by SinYoung Kim with an article on Surgical repair of external inflammatory root resorption with resin-modified glass ionomer cement, At 12 months after surgery, radiographic examination revealed complete healing of the lesion, the gingival attachment was still healthy, and tooth mobility was normal. The patient remained complaint free [16].

\section{Conclusion}

The decision-making process for treatment plan depends on multiple factors according to articles:

1. Tooth structure that remained after an extensive external cervical resorption

2. Capability of adequate primary graft fixation, revascularization

3. Chose the most conservative treatment plan to retain damaged tooth

\section{References}

1. Patel S, Kanagasingam S, Pitt Ford T (2009) External cervical resorption: a review. J Endod 35(5): 616-625.

2. Glendor U (2008) Epidemiology of traumatic dental injuries-a 12 year review of the literature Dental Traumatology 24(6): 603-611. 
3. Andreasen JO, Andreasen FM (2001) Classificacao, etiologia e epidemiologia In: Andreasen JO, Andreasen FM (Eds.), Textbook and Color Atlas of Traumatic Injuries to the Teeth $3^{\text {rd }}$ (Edn.), Artmed, Porto Alegre, pp: 151-180.

4. Turner PJ, Bedi R (1996) Combined orthodontic and restorative management of a case of bilateral ectopic canines and resorbed central incisors. $\mathrm{Br}$ Dent J 180(2): 67-72.

5. Shapira Y, Mischler WA, Kuftinec MM (1982) The displaced mandibular canine. ASDC J Dent Child 49: 362-364.

6. Ruprecht A, Batniji S, El-Neweihi E (1985) The incidence of transposition of teeth in dental patients. J Pedod 9: 244-249.

7. Postlethwaite KM (1989) Resorption of premolar roots by ectopic canines. Br Dent J 167 (11): 397-398.

8. Clarisse $\mathrm{CH} \mathrm{Ng} \mathrm{(2006)} \mathrm{Influence} \mathrm{of} \mathrm{remaining} \mathrm{coronal}$ tooth structure location on the fracture resistance of restored endodontically treated anterior teeth. J Prosthet Dent 95(4): 290-296.

9. Bryan HT (2014) Treatment of a maxillary central incisor with class III invasive cervical resorption and compromised ferrule: A clinical report. J Prosthet Dent 111(5): 356-361.
10. Alves TP (2013) Multidisciplinary approach for the treatment of extensive external cervical resorption after dental trauma. J Operative Dentistry 38(4): 349357.

11. Rhonda R (2002) Restoration and retention of maxillary anteriors with severe root resorption. JADA, 133(1): 67-71.

12. Kim Y, Lee CY, Kim E, Roh BD (2012) Invasive cervical resorption: treatment challenges. Restor Dent Endod 37(4): 228-231.

13. Bartok RI, Vaideanu T, Dimitriu B, Varlan CM, Suciu I, et al. (2012) External radicular resorption: Selected cases and review of the literature. J Med Life 5(2): 145-148.

14. Jiang $\mathrm{YH}$, Lin $\mathrm{Y}$, Jing Ge, Jia-Wei $\mathrm{Z}$, Zhang $\mathrm{L}$, et al. (2014) Multiple idiopathic cervical root resorptions: report of one case with 8 teeth involved successively. Int J Clin Exp Med 7(4): 1155-1159.

15. Leila Eftekhar, Hengameh A, Sanaa J (2017) Management of Invasive Cervical Root Resorption in a Mandibular Canine Using Biodentine as a Restorative Material: A Case Report. IEJ 12(3): 386-389.

16. Kim SY, Yang SE (2011) Surgical repair of external inflammatory root resorption with resin-modified glass ionomer cement. Oral Surg Oral Med Oral Pathol Oral Radiol Endod 111(4): 33-36. 